\title{
Interactive Interface Design based on Social Behaviors of Middle and Aged Women
}

\author{
Wang Jing \\ Wuhan University of Technology, Wuhan 430070, China \\ 591856096@qq.com
}

Keywords: Middle and aged women; Social behavior; Interface design

Abstract: Social nature of people must be decided by people exchanges, to build a variety of relationships and make their own better development and better social integration. Chinese urban elderly are living in reinforced concrete environment, previous neighborhood and kinship is gradual weakening, in relatively simple form of social life, social resources will be relatively scarce, especially the quality of social activity groups of middle and aged women needs to upgrade. This paper analyzes and summarizes the characteristics of social needs and social behavior of groups of older women in Chinese cities, and proposes to improve the social life of interface design principles.

\section{Introduction}

The current social resources are limited in China, the form of relatively are simple, unequal distribution of public facilities for middle and aged women in the way social resources and is relatively scarce, they are eager to social life. But it is not easy to find the emotional compensation by the existing social resources.

\section{Analysis of the Social Behavior of Middle and Aged Female Groups}

\subsection{Social needs of middle and aged women in Chinese cities}

In today's China's national conditions, people's pursuit of physiological needs is no longer in the first place [1]. Middle and aged women in Chinese cities have a stronger sense of emptiness and loneliness because their children are not around because of work or study while they are in a state of retirement or retirement.

According to the different living environment and different levels of education background, the social needs of middle and aged women in Chinese cities are also different. The middle-aged and elderly women with a wide range of personal hobbies and interests have better physical and mental state, healthier mentality and richer social life. Different middle and aged women have different levels of education, resulting in individual differences in their cognitive and learning abilities[2]. The higher the level of education background, the higher the quality requirements of social needs and the wider social sphere. 


\subsection{Social behavior characteristics of middle and aged women in Chinese cities}

\subsubsection{Aggregation}

China's urban middle-aged women grew up in the collectivization of traditional social and cultural background, and under the influence of such a life. In group activities, they can make new friends, get new information, exposure to new things, help resolve solitude brought the children are not around, group activities also can create a new social sphere for the middle-aged women who have conducive to physical and mental health.

\subsubsection{Congregation}

Congregation means that an individual is influenced by the actions or beliefs of others, and changes in personal behavior or beliefs. The herd behavior of middle and aged women in Chinese cities is mainly accepted. In daily life, their dressing style, investment direction, and activities are easily influenced by others.

\section{Design principles for interactive interfaces for middle-aged and female users}

With the increase of age, the functions of various organs of the human body will decline to varying degrees, and the visual acuity, visual search, reaction speed and motion perception ability will also decrease to varying degrees. According to the research, the ability of middle-aged and old people to understand unfamiliar vocabulary has a downward trend with the increase of age[3]. Especially in the face of rapid development of information, middle-aged and elderly people cannot use the previous knowledge system when facing fresh network vocabulary.

\subsection{Analysis of pain spots in the use of mobile phones by middle-aged female users}

\subsubsection{The font is too small to be recognized}

With increasing age, the chances of visual aging of middle and aged women are also increasing by years. Through observation and interviews, some middle and aged women use social software, some people will wear presbyopia glasses, Some people will unconsciously take the phone back in order to see the content more clearly.

\subsubsection{Finger touch screen is easy to delay}

Large-screen mobile phones have better display effects and are more suitable for users with visual aging. However, as the screen of the mobile phone continues to increase, one-handed operation is somewhat difficult. Especially for middle and aged women, their finger flexibility will decrease with age, and the probability of mistakes when performing fine operations will increase. According to the survey data show that the majority of middle-aged female users are accustomed to take his left hand and holding a mobile phone, use the phone right index finger or thumb operation mode, the left hand holding the mobile phone, part of the finger will affect the operation area; controls too close to the edge from the interface, causing a delay circumstances.

\subsubsection{The operation steps are easy to forget}

With increasing age, people's memory declines by years and when faced with complex functions or unfriendly interfaces in smartphone applications, middle and aged people need more time and energy to learn how to operate and use these short-term memories. It becomes a long-term memory, 
and in the case of infrequent use, there is a problem of forgetting the operation steps.

\subsection{Design principles and processes for interactive interfaces for middle and aged women users}

\subsubsection{Structure / function design}

In the design of the interface structure for middle and aged women users, the overall physical structure should not be complicated, the target level should be conciseness, and the operation steps and memory burden of middle and aged women should be reduced. The required information and tools must be placed in appropriate positions. In terms of functional design, it provides simple and clear function navigation when the application is opened, so that the middle and aged female users can roughly understand the main functions in the first use of the application. Allow errors, and give feedback in the first time to inform them of the error to reduce the error rate.

\subsubsection{Information transmission design}

In terms of interface text design, text is an important source of information for middle and aged female users. The size and line spacing of texts greatly affect their efficiency in learning and receiving information. With sans-serif fonts, the sans-serif font will provide better visual contrast with the surface of the object, making it more "clean". Increasing the spacing of the text and increasing the spacing between the words will improve the reading difficulties of middle and aged women. In terms of icon design, use pseudo-objects, image graphics or patterns as much as possible to perform functional metaphors and select objects that are familiar and frequently used in the cognitive habits and cultural levels of middle and aged and people. In terms of feedback information, visual feedback and auditory feedback can be used as the simplest and most direct feedback method.

\subsubsection{Interactive detail design}

In the initial stage of design process, interaction design needs to deeply understand the characteristics and needs of users. What kind of interaction mode can reduce the "lost feeling" when users use it for the first time, predict users to the problems encountered throughout the use process and find corresponding feedback methods to let users solve these problems. Carefully comb the ideas of the interaction process, let the user understand the method used, use the user's language, reduce the use of too professional vocabulary, let the user control the interface, give the user the right to decide freely, and let the user know where they are currently, so that they can make the next decision[4].

In the interactive design process, we should first define the product specific tasks for different tasks, different users have different scenarios, the product of the interaction process may be more than one. After combing the overall tasks and scenarios, find out the interaction points between users and products for different tasks and scenarios. At design time we need to reduce the difficulty of operation, simplify the steps, and use simpler and easier to understand words.

According to research and observation, the most frequently used gestures by middle and aged women are: click, slide, zoom. So we can use the proficient gestures to complete the interactive task.

\subsubsection{Visual design}

In terms of visual design, firstly, we must determine the unity of the interface style, the interface 
design should be easy to understand, avoid using too professional vocabulary. Keep the page function in order, and the text corresponding to the interface function should be easy to understand[5]. When a middle and aged female user has an operational error, be careful to use a friendly reminder to guide the operation steps to avoid fear. Harmony color matching can enrich the design content, increase the layering of the design, and make the interface style uniform[6]. The choice and design of the icon should be consistent with the cognitive level of middle and aged women, using the real world metaphor. In addition, the design of icons, controls and backgrounds must be combined with feminine features, and the use of curves can not only beautify the layout of the interface, but also cause aesthetic resonance of female users.

\section{Summary}

In the face of the increasing attention of middle and aged women, their social behavior and needs also affect our society. How to use design thinking to analyze their personality characteristics and social needs, how to improve their social quality and enrich their social life, this will be a topic worthy of long-term research. In the design area, we want to add more humanized content, and hope that through this research to arouse more attention to the middle and aged women group, I hope that designers will design more practical products for middle and aged women. Let this special group enjoys more convenience.

\section{References}

[1] Zhang Yuming. Study on the Status Quo and Needs of Chinese Women's Old People-Compared with Japan[D].East China Normal University,2008-05

[2] Meng Wei, Tang Zhe. Longitudinal study on the influencing factors of cognitive decline in the elderly [J]. Chinese Journal of Geriatrics, 2000

[3] Xu Shulian. Adult Development Psychology [M]. People's Education Press, 2006

[4] Dan Saffer. Micro-interaction: detail design to achieve excellence products [M]. People's Posts and Telecommunications Press, 2010-10

[5] Peng Jia. Research on interaction design of elderly smart phone APP interface based on user experience [D]. East China University of Science and Technology, 2014

[6] Li Chuanfang, Zheng Yuanxiao. Study on the color discrimination ability of elderly people on computer screen and 100 hue test[J].Science and Technology Journal,2006(01) 